\title{
Progress and challenges in the development of novel implant concepts for cardiovascular, ophthalmologic and otolaryngologic applications
}

Niels Grabow, Institute for Biomedical Engineering, Rostock University Medical Center, Germany

Klaus-Peter Schmitz, Institute for Biomedical Engineering, Rostock University Medical Center and Institute for ImplantTechnology and Biomaterials e.V, Warnemünde, Germany

niels.grabow@uni-rostock.de

Medical device innovations may contribute to the reconstruction of biological functions, and thereby improve the quality of patients' lives, as well as extend their life expectancy. The coordinated research project "RESPONSE - Partnership for Innovation in Implant Technology" (BMBF program Twenty20 - Partnership for Innovation, 2014 - 2021) is focusing on the development of novel concepts for implantable medical devices for cardiovascular, ophthalmologic and otorhinolaryngologic applications. RESPONSE is particularly active in the field of engineering and testing of stentbased implants, as well as local drug delivery coatings for implants. Percutaneous heart valve technology is being researched towards dedicated in vitro testing environments and artificial polymer-based leaflet materials as a replacement for current xenogenic tissue-based materials. Numerical tools are being developed for the structural mechanical and fluid dynamical assessment of implants and implant/host interaction. Employing stents as a platform technology, novel applications are being addressed, such as microstents for glaucoma treatment, as well as stents for the Eustachian tube for treatment of chronic otitis media. The joint research efforts of academia and industry in the RESPONSE consortium address the challenges in implant design, biofunctionalization, process development and production. Particular attention is paid to the process of translation of medical device innovations, cost analysis, as well as health technology assessment. 


\title{
Stenting the Eustachian Tube for Treatment of Chronic Otitis Media
}

\author{
T. Lenarz*, T. Wilfling*, G. Paasche*, K. Schümann**, N. Grabow**, W. Schmidt**, K.P. Schmitz*** \\ H. Müller**** \\ *Department of Otorhinolaryngology, Hannover Medical School, Carl-Neuberg-Str. 1, 30625 Hannover, Germany \\ **Institute for Biomedical Engineering, Rostock University Medical Center, Friedrich-Barnewitz-Str. 4, 18119 \\ Rostock-Warnemünde, Germany \\ ***Institute for Implanttechnology and Biomaterials - IIB e.V., Friedrich-Barnewitz-Str. 4, 18119 Rostock- \\ Warnemünde, Germany \\ **** CORTRONIK GmbH, Friedrich-Barnewitz-Str.4a, 18119 Rostock-Warnemünde, Germany
}

Chronic otitis media affects more than one million people in Germany and is the main cause of permanent conductive hearing loss. Key in pathophysiology is the impairment of the Eustachian tube function and non-sufficient ventilation of the middle ear. Current treatment options including grommets or balloon dilatation are not sufficient to successfully address the underlying pathology. Stenting the tube is an innovative concept to restore the impaired tube function. The stent is placed in the cartilaginous part of the Eustachian tube in order to provide sufficient ventilation but avoiding patulous tube is permanent opening. Permanent and resorbable stents are designed.

Current status of development:

Dimensions of the human tube have been determined by cadaver experiments. Still in different stent-designs we right from cardiovascular stents could be placed successfully in the tube using specifically developed surgeon system. Invivo tests were done in the black sheep animal model. Over a period of 6 month the Eustachian tube could stay open with a minimum of tissue reaction. The function of the tube was unimpaired.

Final design and development of a human prototype stent system using nitinol and polymer stents. 


\section{Electrospinning for polymeric implants in cardiovascular applications Accelerated Degradation of Electrospun Poly(L-lactide) Scaffolds}

Stefanie Kohse: University Medical Center Rostock, Institute for Biomedical Engineering, Friedrich-Barnewitz-Str. 4, D-18119 Rostock, Germany, e-mail: stefanie.kohse@uni-rostock.de

Daniela Arbeiter, Thomas Reske, Thomas Eickner, Niels Grabow: University Medical Center Rostock, Institute for Biomedical Engineering, Rostock, Germany, e-mail: daniela.arbeiter@uni-rostock.de, thomas.reske@uni-rostock.de; thomas.eickner@uni-rostock.de, niels.grabow@uni-rostock.de

Klaus-Peter Schmitz: University Medical Center Rostock, Institute for Biomedical Engineering, Rostock, Germany and Institute for Implant Technology and Biomaterials e.V., Rostock, Germany, e-mail: klaus-peter.schmitz@uni-rostock.de Michael Stiehm: Institute for Implant Technology and Biomaterials e.V., Rostock Germany, e-mail: michael.stiehm@uni-rostock.de

The possibility for replacement of certain implant materials by polymers is an appealing alternative because of their simple production, high availability and versatility for tailoring desired properties. [1] In this context, innovative process technologies generate new impulses for future implant developments. Electrospinning is a straightforward method producing fibrous polymer scaffolds that can be applied in drug delivery systems as well as for polymer-based implants. Concerning the use of biodegradable polymer nonwoven scaffolds for cardiac tissue engineering the rate of degradation is one of the key parameters for a successful deployment. Performance of accelerated hydrolytic degradation is of high interest in recent research to make fast predictions for different materials in comparison. In this study we present accelerated degradation of poly(L-lactide) nonwovens scaffolds. For evaluation of degradation process mass loss, change in molecular mass, surface morphology and thermal behavior are investigated. Successful nonwoven degradation within 2 weeks was achieved using alkaline conditions for hydrolysis. The results show a rapid mass loss up to $21.4 \%$ of the initial weight and a loss of molecular weight up to $64 \%$ concerning $\mathbf{M}_{\mathrm{w}}$. Thermal behavior and surface morphology show initial degradation of amorphous regions of polymer. Further studies comparing different semi-crystalline materials and surface structures will be performed.

[1] W. Khan, E. Muntimadugu, M. Jaffe, A.J. Domb, Advances in Delivery Science and Technology - Focal Controlled Drug Delivery: Chapter 2. Implantable Medical Devices, CRS - Springer Publications, 2014. 


\section{Drug release systems based on hyaluronan materials}

Matthias Schnabelrauch, INNOVENT e.V., Biomaterials Department, Jena, Germany, ms@innovent-jena.de Ralf Wyrwa, INNOVENT e.V., Biomaterials Department, Jena, Germany, rw1@innovent-jena.de Torsten Walter, INNOVENT e.V., Biomaterials Department, Jena, Germany, tw@innovent-jena.de

Hyaluronan is a high-molecular weight, linear glycosaminoglycan (GAG) and part of the extracellular matrix. It can be found in connective, epithelial and neural tissues and delivers mechanical stability, acts as water reservoir and lubricant and plays a multifaceted role in skin wound healing processes.

Chemically modified hyaluronan derivatives bearing cross-linkable functional groups, e. g. (meth)acryl-functions can form stable hydrogels upon crosslinking. Depending on the degree of substitution of (meth)acrylated hyaluronan and GAG concentration three-dimensional hydrogels with tuneable mechanical properties and degradation behaviour can be obtained using photochemical crosslinking. These gel-like materials exhibit great potential for various medical applications.

Hyaluronan, especially one with exceptionally high molecular weight, shows great ability to produce ultrathin fibers in electrospinning processes forming non-woven fleeces with high surface to weight ratio.

Various ophthalmic drugs can be integrated in such hydrogels and electrospun fleeces showing the necessary mechanical properties (softness) for applications in the eye. In our studies the beta blocker timolol was incorporated in both types of hyaluronan materials. We were interested to develop novel drug delivery systems with sustained and controlled release usable in ophthalmological applications (glaucoma therapy). We investigated different strategies of timolol integration in hydrogels and electrospun fibers based on ionic interactions or covalent attachment of suitable timolol prodrugs. To stabilize hyaluronan carriers against aqueous surroundings various in-process and post-process treatment methods were tested. The degradation behavior of our hyaluronan systems is tuneable from few days to several months and can therefore be adapted to the need of applications. The release profiles of timolol from the generated drug delivery systems were investigated showing significant differences depending of the used structure, mode of drug incorporation and processing method emphasizing such hyaluronan based materials for the development of well adapted application specific drug delivery systems. 


\section{Regenerative cardiac valve prostheses based on cell seeding of polymeric scaf- folds}

Tobias Schilling, Department of Cardiothoracic, Transplantation, and Vascular Surgery, Hannover Medical School, Hannover, Germany, schilling.tobias@mh-hannover.de

The replacement of diseased heart valves represents one of the most common fields of application of cardiovascular surgery. However, current therapeutic options suffer from severe drawbacks such as the lack of regenerative capabilities of heart valve prostheses. Nevertheless, tissue engineering allows for the development of viable implants, with a regenerative and growth potential after implantation in the patient. Since xenogeneic starter matrices still trigger a severe immune reaction of the host versus the graft, and since allogeneic human substrates are not available in sufficient numbers, we focus on degradable and non-degradable electrospun polymeric fleeces to engineer regenerative cardiac valve prostheses.

However, in the past the main issues of synthetic and natural polymeric heart valves were low durability, fast degradation rate, poor long-term survival, and thrombotic and calcific degeneration of the valve leaflets, leading to severe complications. Thus, implantation of these valves is still not an option in the clinical setting. Currently, no optimal polymer for manufacturing an ideal cardiovascular prosthesis has been identified yet. Hence, we developed a bioreactor system to mimic a physiological environment for cell seeding of the acellular polymeric nanofiber fleeces in terms of tissue engineering. A confluent endothelial cell layer would prevent the immune reaction of the host and would eventually allow for a slow physiological remodelling of such a natural-synthetic hybrid graft. Histological investigations and scanning electron microscopy are used to assess the efficacy of the seeding process.

Further investigations such as testing the seeded cells survivability of crimping procedures to prepare for catheter-based implantation and in vivo assessment of the degradation rate of the polymeric scaffold and the host's immune reaction in a small animal model are currently being prepared. 


\title{
Clinical needs and perspectives for the development of new generation TAVR
}

\author{
Öner A, Kaule S, Grabow N, Schmitz KP, Stiehm M
}

Alper Öner, Heart Center, Department of Cardiology, Rostock University Medical Center, Rostock, Germany Klaus-Peter Schmitz and Michael Stiehm, Institute for ImplantTechnology and Biomaterials e.V., RostockWarnemünde, Germany

Niels Grabow, Klaus-Peter Schmitz, Institute for Biomedical Engineering, Rostock University Medical Center, Rostock-Warnemünde, Germany

Transcatheter aortic valve replacement (TAVR) has become the treatment of choice for inoperable or high-risk patients with symptomatic aortic stenosis. Since clinical performance of established transcatheter aortic valve prostheses (TAVP) is constantly increasing, ongoing studies aim for an extension of the therapy from high-risk to intermediate and subsequently low-risk patients. Ongoing research in the field of TAVR focuses on providing TAVR-procedures for younger patients. Therefore, TAVP durability is becoming one of the key aspects for future TAVP generations with respect to new valve materials as well as structural and fluid mechanically optimized valve body and TAVP designs. Noncalcification, inhibited inflammation and promoted endothelialization are further features of novel leaflet materials. The reduction of the peacemaker rate addresses the stent design due to the radial strength and in addition, new skirt configurations are needed to compensate individual pathophysiological annulus geometries due to prevent paravalvular leakage. Furthermore, it is well accepted that the arrangement of the Valsalva sinus and the valve leaflets influences the hemodynamic situation and plays a important role for the washout of the sinus and the blood supply of the coronaries in healthy individuals. Novel valve designs should take hemodynamic aspects aspects, especially the vortex formation in the Valsalva sinus, into account. Moreover, future catheter systems should enable a controlled implantation regarding valve depth and circumferential positioning. Improved visibility of the prosthesis as well as implantation features such as re-sheathabilty help the cardiologists reducing the procedural risks.

Future high-resolution imaging techniques in conjunction with highly tactil catheter systems and hemodynamic optimized valve designs with highly durable materials could potentially provide a desired lifelong implant even for younger patients. 


\section{Development of model drug-eluting implants via fused deposition modeling}

Anne Seidlitz, Institute of Pharmacy, Center of Drug Absorption and Transport, EMA University of Greifswald, 17487 Greifswald, Germany, anne.seidlitz@uni-greifswald.de

Wiebke Kempin, Institute of Pharmacy, Center of Drug Absorption and Transport, EMA University of Greifswald, 17487 Greifswald, Germany, wiebke.kempin@uni-greifswald.de

Werner Weitschies, Institute of Pharmacy, Center of Drug Absorption and Transport, EMA University of Greifswald, 17487 Greifswald, Germany, werner.weitschies@uni-greifswald.de

Additive manufacturing techniques are of rising interest in the pharmaceutical and biomedical field, especially with regard to treatment options optimized for small groups of patients or individual patients. The degree of personalization may include patient-specific shape, for example to provide exact fit into body conduits, as well as individually adapted drugs, combinations of drugs, drug doses and / or drug releases as well as other factors. If these concepts are combined, a necessity for a large product portfolio arises which cannot be fulfilled using classical manufacturing techniques.

Fused deposition modeling is a technique which is very promising with regard to the aim of fabricating patient-specific implants. In brief, a filament, typically consisting of a thermoplastic polymer and possibly a drug as well as other excipients, is molten inside the hot-end of a nozzle and layerwise deposited according to a computer-aided design to built the desired implant. Alternatively semi-solid material of a suitable viscosity may be extruded with a slightly different setup and also be layerwise depostited followed by a hardening step (e.g. drying, cross-linking, etc.). However, drug-loaded filaments or even medical grade drug-free filaments or pastes for extrusion are not comerically available.

Filaments composed of pharmaceutically used polymers and model drugs were produced via hot-melt extrusions and tested regarding their printability. Filaments that were successfully printed were also evaluated regarding their drug release behaviour. Using this approach a wide range of release profiles ranging from minutes to months were obtained in dependency of the used polymers, the solubility of the model drug in the polymers and the implant size. By using a paste extrusion approach flexible implants composed of gelatin were also produced futher underlining the versatility of the fused deposition modelling approach for the manufacturing of individualized implants. 


\section{Optimization of stent designs regarding the thrombosis risk using computational fluid dynamics}

Carolin Wüstenhagen, Institute for ImplantTechnology and Biomaterials e.V., Friedrich-Barnewitz-Str. 4, 18119 Rostock-Warnemünde, Germany, e-mail: carolin.wuestenhagen@uni-rostock.de

Niels Grabow, Institute for Biomedical Engineering, Rostock University Medical Center, Friedrich-Barnewitz-Str. 4, 18119 Rostock-Warnemünde, Germany, e-mail: niels.grabow@uni-rostock.de

Klaus-Peter Schmitz, Institute for ImplantTechnology and Biomaterials e.V. and Institute for Biomedical Engineering, Rostock University Medical Center, Friedrich-Barnewitz-Str. 4, 18119 Rostock-Warnemünde, Germany, e-mail: klauspeter.schmitz@uni-rostock.de

Michael Stiehm, Institute for ImplantTechnology and Biomaterials e.V., Friedrich-Barnewitz-Str. 4, 18119 RostockWarnemünde, Germany, e-mail: michael.stiehm@uni-rostock.de

In-stent thrombosis is a major complication of stent implantations. Unlike pathological occurrences as in stent restenosis for instance, thrombosis represents an acute event associated with high mortality rates. Experiments show that low wall shear stress promotes undirected endothelial cell coverage of the vessel wall and therefore increases the risk of thrombus formation. Stent design represents a crucial factor influencing the surface areas of low wall shear stress and thus the incidence of acute in stent thrombosis.

In this study, we present an optimization method for stent designs with minimized thrombosis risk. A generic stent design was developed, based on five different stent parameters. Optimization was conducted based on computational fluid dynamics analysis and the gradient free Nelder-Mead-Method. For each optimization step, a numerical fluid simulation was performed in a vessel with a reference vessel diameter of $2.70 \mathrm{~mm}$ with stent overexpansion ratio of 1.0:1.1. For each numerical fluid simulation a physiological Reynolds number of 250, resulting in a mean velocity of $0.331 \mathrm{~m} / \mathrm{s}$ at the inlet and a laminar flow as well as stiff vessel walls are assumed.

The impact of different stent designs was analyzed based on the wall shear stress distribution. As a basis for the comparison of different stent designs, a dimensionless thrombosis risk number was calculated from the area of low wall shear stress and the overall stented area. The first two optimization steps already provide a decrease of thrombosis risk of approximately $83 \%$. In conclusion, computational fluid dynamic analyses and optimization methods represent a useful tool for the development of hemodynamically optimized stent designs with minimized thrombosis risk. 


\section{Inflammation-responsive polymer conjugates for adaptive delivery of anti- inflammatory compounds}

Manfred F. Maitz, Leibniz Institut für Polymerforschung Dresden, Dresden, Germany, maitz@ipfdd.de Tina Helmecke, Carsten Werner, Leibniz Institut für Polymerforschung Dresden, Dresden, Germany

Blood coagulation and inflammation due to innate immune responses are persisting problems in the application of cardiovascular devices, such as vascular stents. Anticoagulant and anti-proliferative surface modification is commonly applied to prevent consequent thrombosis or restenosis. However, lacking control over the activity limits the efficacy of these approaches and impedes physiological regeneration.

Herein, we present a feedback-controlled release system, based on four-armed, end-functionalized poly(ethylene glycol) (PEG) conjugated with heparin and anti-inflammatory molecules via selective protease-cleavable linker peptides. Leukocyte elastase, released during inflammation from activated granulocytes, serves as a trigger for cleavage of the linker peptide resulting in the degradation of the hydrogel. In consequence, the hydrogel releases its cargo of antiinflammatory substances along with anti-coagulant heparin in response to pro-inflammatory conditions. As antiinflammatory substance, the complement receptor blocker PMX53 has been linked covalently to the hydrogel via the cleavable peptide linker. It is shown that the bioactivity in this conjugation is maintained and the inhibitor is cleaved off by elastase in a dose-dependent manner. PMX53 containing gels were observed to downregulate granulocyte activation in an in vitro inflammation model. The modularity of the system allows for the (optionally combined) release of other anti-inflammatory molecules like cyclosporine derivatives or the complement inhibitor compstatin.

In sum, the presented hydrogel system offers unprecedented options to address important clinical needs by the inflammation-controlled release of anti-inflammatory substances. 